\title{
Lacrimal Gland Carcinoma pT2b TNM Finding v8
}

National Cancer Institute

\section{Source}

National Cancer Institute. Lacrimal Gland Carcinoma pT 2b TNM Finding v8. NCI

Thesaurus. Code C140790.

Lacrimal gland carcinoma with tumor measuring more than $2 \mathrm{~cm}$ but $4 \mathrm{~cm}$ or less in greatest dimension with periosteal involvement only. (from AJCC 8th Ed.) 